\title{
Chitosan and oligo(DL-lactic acid) networks: Correlations between physical properties and macromolecular configuration
}

\author{
Claudio B. Ciulik a , Oigres D. Bernardinelli ${ }^{\text {b }}$, Eduardo R. de Azevedo ${ }^{\text {b }}$, Leni Akcelrud ${ }^{\text {a, * }}$ \\ a Paulo Scarpa Polymer Laboratory - LaPPS, Federal University of Paraná - UFPR, P.O. Box 19081, 81.531-990, Curitiba, Paraná, Brazil \\ ${ }^{\mathrm{b}}$ Physics Institute of São Carlos, São Paulo State University - USP, P.O. Box 369, 13560-970, São Carlos, São Paulo, Brazil
}

\section{A R T I C L E I N F O}

\section{Article history:}

Received 23 February 2016

Received in revised form

12 April 2016

Accepted 13 April 2016

Available online 14 April 2016

\section{Keywords:}

Chitosan

Oligo(DL-Lactic acid)

Networks

\begin{abstract}
A B S T R A C T
Aiming the obtainment of materials with useful properties for bio-applications, as thermal and mechanical (in particular flexibility), chitosan and low molecular weight poly(lactic acid) were chosen due to their biodegradability and biocompatibility in various bio-environments. Networks formed by oligo(DL-lactic acid) segments linked to the chitosan chains were prepared, in which the ratio between the components varied systematically. A thorough structural characterization was performed, including the degree of crosslinking and the size of the crosslinking blocks. The thermal and mechanical properties varied according the ratio between the components in a non-linear way, which was discussed in terms of the macromolecular architecture. The data were compared with previous results obtained for grafts with the same composition aiming to verify the effect of the macromolecular architecture on the macroscopic properties.
\end{abstract}

(๑) 2016 Elsevier Ltd. All rights reserved.

\section{Introduction}

The combination of important properties found in natural polymers, such as non-toxicity, biodegradability and biocompatibility have sparked the interest on this kind of material for biomedical and biotechnology applications in the last decades [1-6]. However, they often lack the adequate mechanical properties needed for those specific purposes, as opposed to synthetic polymers that have suitable physical properties but limited biocompatibility [7-11]. Aiming to circumvent this problem, we have studied the combination of chitin with a biodegradable polyurethane, in the form of blends [12] and of networks with various compositions and crosslinking densities [13]. The potential application of the two systems as biomedical materials was also studied, showing promising results [14]. Aiming to get further improvement either in mechanical as in bio-properties, a new combination was explored: chitosan with poly(lactic acid) in the following macromolecular architectures: in the first, oligo(DL-lactic acid) segments, with systematic variation in the size and distance among each other were grafted onto the chitosan backbone [15]. In the second, which is the subject of the present contribution,

\footnotetext{
* Corresponding author. Federal University of Paraná, Chemistry Department, P.O. Box 19081, 81.531-990, Curitiba, Paraná, Brazil.

E-mail address: akleniak@gmail.com (L. Akcelrud).
}

networks of the two components were studied. The schematic chemical representation of a generic network is illustrated in Fig. 1. The rationale of this approach is based on the biocompatibility of the poly(DL-lactic acid) and on the possibility of variation in the degree of crosslinking which could afford permeation selectivity for drug delivery applications. The preparation and characterization (molecular, thermal and mechanical) are described, discussed and compared with the grafted copolymers of same composition.

\section{Experimental}

\subsection{Materials}

Chitosan (low molecular weight, degree of deacetylation 75-85\% given by the supplier) was purchased from Sigma-Aldrich (China). DL-lactic acid 90\% was purchased from Fluka Analitical (Japan). Glacial acetic acid was purchased from Vetec (Brazil). Metanol and acetone were obtained from Synth (Brazil). Chloroform was obtained from Biotec (Brazil). All solvents were PA grade and were used without further treatment.

\subsection{Methods}

\subsubsection{Synthesis of $N$-succinyl chitosan}

Chitosan was succinylated according to a previously reported 


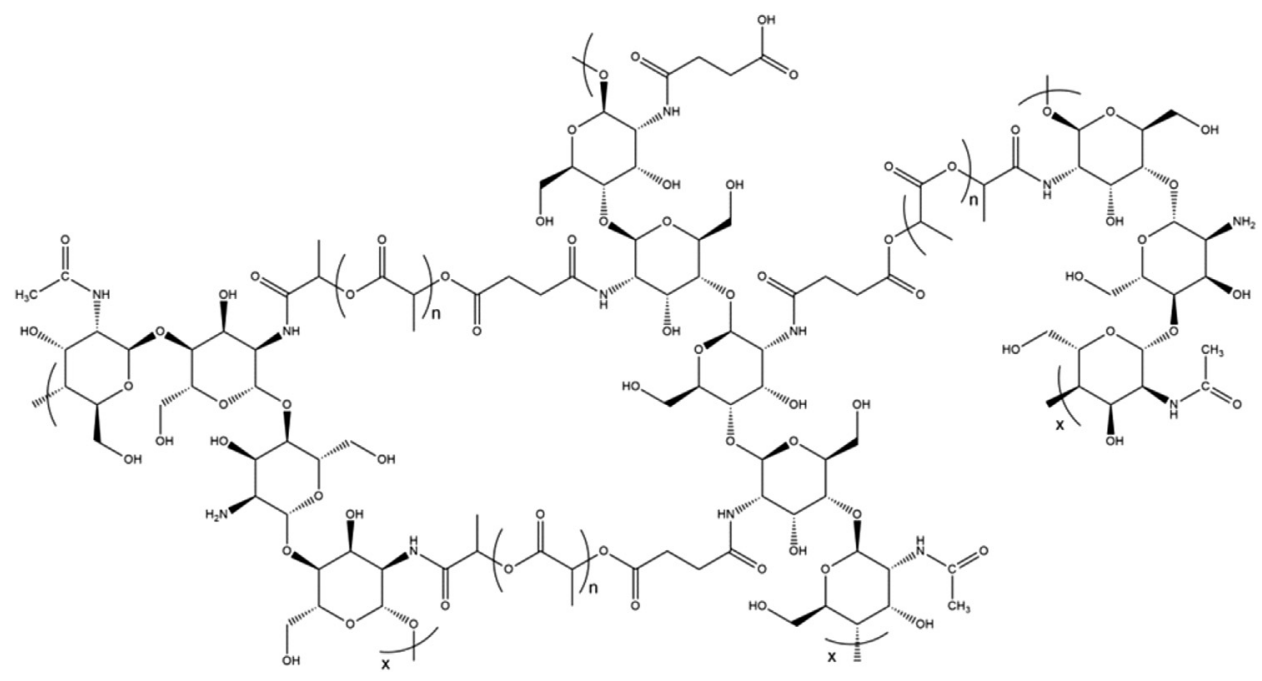

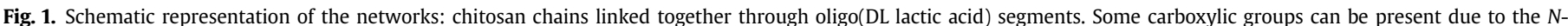
succinyl chitosan derivative used in the network preparation.

method [16] with some modifications. Briefly, $1 \mathrm{~g}(6.21 \mathrm{mmol})$ of chitosan was dissolved under stirring in a $1 \%(\mathrm{v} / \mathrm{v})$ aqueous acetic acid solution $(70 \mathrm{~mL})$. Then $4.22 \mathrm{~g}(42.8 \mathrm{mmol})$ of succinic anhydride, previously dissolved in $30 \mathrm{~mL}$ of acetone, was added drop wise to the polymer solution. The reaction was maintained under stirring overnight at room temperature. The obtained viscous solution was precipitated in acetone, filtered to remove the solvent and then washed with aqueous $70 \%$ acetone and pure acetone respectively. Finally, the product was dried at $40{ }^{\circ} \mathrm{C}$ under vacuum for $24 \mathrm{~h}$.

2.2.2. Synthesis of polymer networks (reaction of chitosan with $\mathrm{N}$ succinyl chitosan and oligo(DL-lactic acid)

$0.81 \mathrm{~g}$ ( $3.10 \mathrm{mmol})$ of $\mathrm{N}$-succinyl chitosan was dissolved in $20 \mathrm{~mL}$ of distilled water and reserved (this solution was labeled as solution A). $0.5 \mathrm{~g}$ (3.10 mmol) of chitosan were dispersed in $20 \mathrm{~mL}$ of distilled water and then added to pre-determined amounts of DLlactic acid ( $\mathrm{pH}$ around 3). This solution (labeled as B) was stirred until completely dissolution of chitosan. Solution A was slowly added to solution $\mathrm{B}$, and the final solution was maintained under stirring at $60{ }^{\circ} \mathrm{C}$ for $8 \mathrm{~h}$. The formation of polymer networks occurred after the reaction medium was poured into Petri plates and kept at $90{ }^{\circ} \mathrm{C}$ under vacuum during $6 \mathrm{~h}$ for film formation. All samples were purified via Soxhlet extraction with methanol and chloroform, separately, for $24 \mathrm{~h}$.

The networks were labeled as CR01 to CR06, the former with the lowest and the latter with the highest OLA contents.

\subsection{Characterization}

${ }^{1} \mathrm{H}$ NMR spectra of $\mathrm{N}$-succinyl chitosan was acquired in $\mathrm{D}_{2} \mathrm{O}$ on a Bruker AVANCE 400 spectrometer, operating at $9.4 \mathrm{~T}, 400.13 \mathrm{MHz}$, equipped with a multinuclear probe of $5 \mathrm{~mm}$. Recycle delay of $100 \mathrm{~s}$ was used.

High Resolution Solid-State NMR (SSNMR) experiments were performed using a VARIAN INOVA spectrometer operating at ${ }^{13} \mathrm{C}$ and ${ }^{1} \mathrm{H}$ frequencies of 88.02 and $350.50 \mathrm{MHz}$. A VARIAN 7-mm Magic Angle Spinning (MAS) double-resonance probe head was used at spinning frequency of $4.5 \mathrm{kHz}$ controlled by a VARIAN pneumatic system that ensures a rotation stability of $\pm 2 \mathrm{~Hz}$. Typical $\pi / 2$ pulse lengths of $5.0 \mu$ s were applied to both ${ }^{13} \mathrm{C}$ and ${ }^{1} \mathrm{H}$. To achieve dead-time-free detection all spectra were recorded with a
Hahn spin echo generated by an $180^{\circ}$ pulse applied one rotation period $\left(t_{r}\right)$ after the end of excitation. Time proportional phase modulated (TPPM) proton decoupling with field strength of $60 \mathrm{kHz}$ was used in all experiments. The ramp for Cross-Polarization (CP) was implemented with an amplitude increment from 90 to $100 \%$. ${ }^{13} \mathrm{C}$ Multiple CP under MAS (MultiCP) experiments [17] were performed using nine periods of cross-polarization, with time of $1 \mathrm{~ms}$, spaced by the repolarization period tz of $0.9 \mathrm{~s}$, except for a last $\mathrm{CP}$ period of $0.8 \mathrm{~ms}$. The recycle delays were $2 \mathrm{~s}$. MultiCP is a recently proposed method that can provide quantitative ${ }^{13} \mathrm{C}$ nuclear magnetic resonance spectra (NMR) with higher signal-to-noise ratios, in a short period of the time compared with the standard quantitative direct-polarization NMR. However, to guarantee that Multi$\mathrm{CP}$ provided quantitative spectra one needs to make sure that there is not molecular motion on the frequency scale of $(10-100 \mathrm{kHz})$, which interferes with the cross-polarization and high power decoupling and brings about a motion induced signal decay. This was checked in each analyzed sample using ${ }^{1} \mathrm{H}$ broad band NMR, which showed that there is no motion in this frequency range at room temperature. For checking the choice of parameters in MultiCP, the spectra obtained using MultiCP and single pulse excitation (SPE) with long recycle delay were compared for the sample CR01, the one with the lowest OLA content. The spectra obtained by both excitation methods were virtually the same. More details on the method along with the pulse sequence can be found in Ref. [17].

Dynamic mechanical analyses were performed in a Netzsch DMA 242C equipment with an amplitude of $10 \mu \mathrm{m}$, from $-150^{\circ} \mathrm{C}$ to $200{ }^{\circ} \mathrm{C}$ at a heating rate of $3 \mathrm{~K} / \mathrm{min}$ at different frequencies, in single cantilever bending mode using a material pocket.

Tensile measurements were performed using dumbbell shaped specimens, in a INSTRON machine 4467 model, equipped with a $100 \mathrm{Kgf}$ cell and $\mathrm{L}_{0}$ (initial length) of $10 \mathrm{~mm}$. The speed was of $10 \mathrm{~mm} / \mathrm{min}$.

\section{Results and discussion}

\subsection{Synthesis of N-succinyl chitosan}

For the obtainment of the chitosan-OLA networks, the chemical modification of one of the two components was needed, since the system lacked the required functionalities for the linking between 
the chains. Regarding this issue, several references concerning the chemical modification of chitosan can be found in the literature, for various purposes [18-23]. One possibility consisted in react the end hydroxyl group of OLA chain with carboxyl groups, which could be provided by reacting the OLA with the succinic anhydride. The networks would then be formed through the esterification of the resulting carboxyl groups of OLA with the hydroxyl ones of the chitosan. That route however, requires an excess of succinic anhydride, which would lead to the acid catalyzed OLA depolymerization $[24,25]$. Due to this problem, the route chosen was based on two steps. In the first, chitosan reacts with succinic anhydride resulting in $\mathrm{N}$-succinyl chitosan, as shown in Fig. 2. In the second, a mixture of chitosan and $\mathrm{N}$-succinyl chitosan reacts with DL-lactic acid. In this step two types of chemical reactions took place: amidation (amino groups of chitosan reacting with the carboxyl ones of lactic acid) and esterification (carboxyl groups of $\mathrm{N}$-succinyl chitosan reacting with the hydroxyl ones of lactic acid). This set of concomitant reactions is best visualized by inspection of Fig. 3. The possible chemical interactions in this mixture are the protonation of the amino groups of chitosan by DL-lactic acid, followed by amide formation, and by the reaction of the carboxyl groups of $N$-succinyl chitosan with the hydroxyl ones of the DL-lactic acid. In other words, the role of DL-lactic acid as a crosslinking agent can be described as follows: its hydroxyl groups react with the carboxyl ones of $\mathrm{N}$ succinyl chitosan while its carboxyl groups react with the amino ones of the original chitosan. Since lactic acid is di-functional, it could undergo polymerization, tying together the two ends of the OLA segment, thus forming the network system, as shown in Fig. 3.

\subsection{Quantitative determination of the degree of conversion of chitosan to N-succinyl chitosan by ${ }^{1} \mathrm{H} N \mathrm{NMR}$}

The quantitative determination of the degree of conversion of chitosan to $N$-succinyl chitosan, as illustrated in Fig. 4, was performed using ${ }^{1} \mathrm{H}$ NMR. The spectrum of the obtained substituted polymer is shown, and the assignment of the signals, based on reference [26] are displayed in Table 1.

The observation of the structure depicted in Table 1 leads one to expect that the ratio of the integration of the signals related to the hydrogen atoms $8,8^{\prime}$ (two $\mathrm{CH}_{2}$ groups from succinyl units) and to 2 ( $\mathrm{CH}$ groups of both acetylated and non acetylated chitosan units, linked to $\mathrm{N}$ ), to be equal to four times the ratio between the number of succinyl units and the number of chitosan (acetylated + non acetylated) units. This ratio was found to be $(4.0 \pm 0.1)$, with the error calculated based on the spectrum signal to noise. This result indicates that within the experimental error, all of the available amino groups of the original chitosan reacted with succinic anhydride, resulting in the amide bond formation.

\subsection{Network formation (CR)}

The most probable mechanism involves the formation of an intermediary state in the form of a lactate salt at the amino sites of the chitosan chain, as illustrated in Fig. 5, while parallel reaction between $N$-succinyl chitosan and hydroxyl group of DL-lactic acid occurs. The same molar ratio of $\mathrm{N}$-succinyl chitosan and chitosan was used in each reaction, whereas the proportion of DL-lactic acid varied systematically, to provide various degrees of crosslinking and various lengths of interchain OLA segments.

As mentioned, the networks were labeled as CR01 to CR06, the former with the lowest and the latter with the highest OLA contents. The crosslinking degree of the networks was set by varying the ratio chitosan $+N$-succinyl chitosan/DL-lactic acid. The greater the amount of $\alpha$-hydroxy acid, the higher the crosslinking degree expected, due to high availability of the monomer in the reaction medium.

\subsection{Structural characterization of networks by SSNMR}

The sketch of the chemical structure of the networks system, (CR materials), is shown in Fig. 6a. Fig. 6b presents a comparison between MultiCP spectra of CR01 and CR06 samples. The signal assignments based on literature data [21] are depicted according to the carbon numbering shown in Fig. 6a. The signals in the 50-110 ppm region are consistent with the chitosan/chitin backbone [27], except for the shoulder at $61.6 \mathrm{ppm}$ (dashed green line), which is related to $\mathrm{O}-\mathrm{C}-\mathrm{H}$ carbons ( $\mathrm{C} 10)$ from OLA groups [15]. The signals at $16.6 \mathrm{ppm}$ and $20.6 \mathrm{ppm}$ are typical of $\mathrm{CH}_{3}$ carbons from respectively, chitin (C8) and OLA (C11) units, while the signal at $\sim 30 \mathrm{ppm}$ is relative to $\mathrm{CH}_{2}$ carbons of succinyl units (C12 and $\mathrm{C} 13$ ), indicated by the red bracket. The signal at $\sim 175 \mathrm{ppm}$ is due to $\mathrm{C}=\mathrm{O}$ groups (C7, C9, C9').

Fig. 7a shows the spectra obtained for samples with CR01 to CR06. All spectra were normalized by the integral of the corresponding C1 signal. Therefore, the increase in the intensities of C10 and $\mathrm{C} 11$ signals indicated by the dashed green lines, are related to the increase in the number of OLA units in the sample. For sample CR06, it is also possible to observe the appearance of a narrower signal in the region attributed to $\mathrm{C} 12$ and $\mathrm{C} 13$. Line narrowing in NMR of organic solids can be attributed to both the presence of molecular motions with rates $>10 \mathrm{kHz}[28,29]$ or increase of local conformational order [30]. ${ }^{1} \mathrm{H}$ time-domain NMR measurements (not shown) revealed that there is no molecular motion in such a frequency scale in the $\mathrm{CR}$ materials. Thus, the narrower C12,13 lines is possibly related to the crosslinking of the succinyl group onto chitosan, which induces a conformational restriction due to binding. The increase in the amount of crosslinked chains is also supported by the change in shape of the lines corresponding to C3 and C5; see gray dashed lines in Fig. 6b. According to reference [27], the



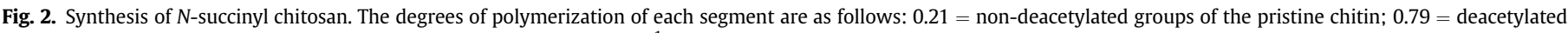
groups, $\mathrm{Y}=$ non reacted amine groups of chitosan ( 0.02 determined by ${ }^{1} \mathrm{H}$ NMR) and $\mathrm{X}=$ succinylated groups. 


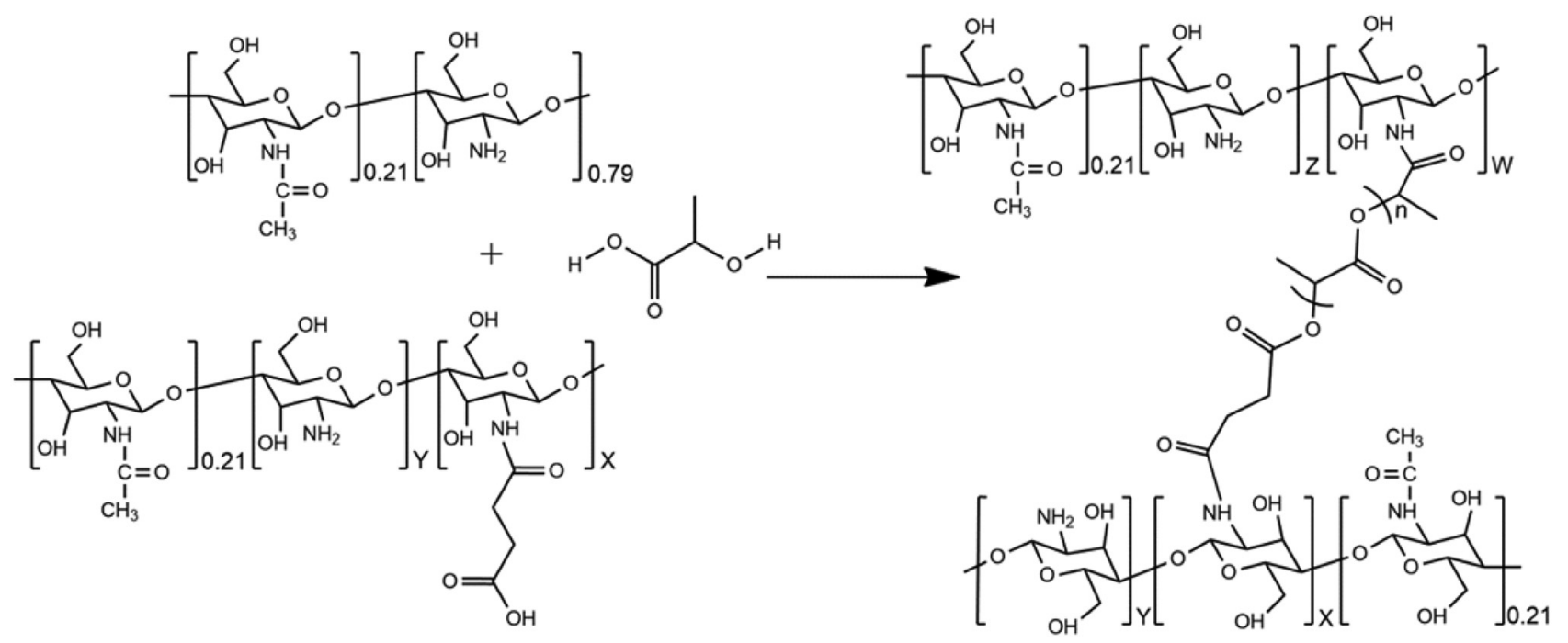

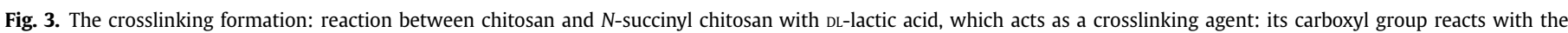
amino group of chitosan and its hydroxyl group reacts with the carboxyl ones of $\mathrm{N}$-succinyl chitosan.



Fig. 4. ${ }^{1} \mathrm{H}$ NMR spectrum of $\mathrm{N}$-succinyl chitosan. be made based on the ${ }^{13} \mathrm{C}$ MultiCP experiments [17] depicted in Fig. 7b. Although the strong line superposition in the $50-90 \mathrm{ppm}$ region prevents the use of these signals for such analysis, the 10-40 ppm spectral region can be reliably fitted into four Gaussian lines, as exemplified in Fig. 7b. These lines are associated to $\mathrm{CH}_{3}$ carbons of the acetylglucosamine (blue) and OLA (green) units and $\mathrm{CH}_{2}$ carbons in crosslinked and non crosslinked (red) succinyl units. All spectra were normalized by the integral of signal $\mathrm{C} 1$, so the area under each Gaussian line (intensity) is proportional to the amount of the corresponding segments relative to the total number of glucosamine + acetyl glucosamine units. The fitted spectra show that the intensity of the C8 signal is constant in all spectra, suggesting that the number of acetyl glucosamine units remains constant. The number of OLA units increases from CR01 to CR06, as shown by the intensities of the C11 signals in the corresponding spectra. In addition, the integral intensity over both succinyl sig-

Table 1

Assignment of the ${ }^{1} \mathrm{H}$ NMR signals of $N$-succinyl chitosan.

\begin{tabular}{llll}
\hline $\mathrm{N}^{\circ}$ & Chemical shift $(\mathrm{ppm})$ \\
1 & 4.6 & 3.1 & $3.6-3.9$ \\
$3,4,5,6$ & 2.0 & 2.5 &
\end{tabular}

replacement of the chitosan $\mathrm{NH}_{2}$ group by a $\mathrm{NH}$ group produces a down field shift in the $\mathrm{C} 3$ signal (dashed black lines). Since in the CR samples the signals corresponding to $\mathrm{C} 3$ and $\mathrm{C} 5$ appear superposed in line 5 , this is seen as a progressive change in the line shape as more $\mathrm{NH}_{2}$ groups are replaced by $\mathrm{NH}$, that is, the crosslinking degree increases. All these features are observed in Fig. 7a for samples CR02, CR03, CR04, CR05, CR06, pointing to an increasing amount of crosslinked units as a function of the OLA concentration.

Besides the qualitative discussions, quantitative estimations can nals, red Gaussians, turn to be constant for all samples, which agree with the constant amount of succinyl in all samples. More interesting is that the integral intensity of the signal attributed to crosslinked succinyl units (signal between 30 and $40 \mathrm{ppm}$ ) clearly increase from CR01 to CR06, while the signal from non crosslinked succinyl diminishes. This evidences that the amount of crosslinked succinyl groups increases as a function of the OLA addition, while the total number of succinyl groups remains constant. Thus, taking the ratio between the integral over the signal due to crosslinked 


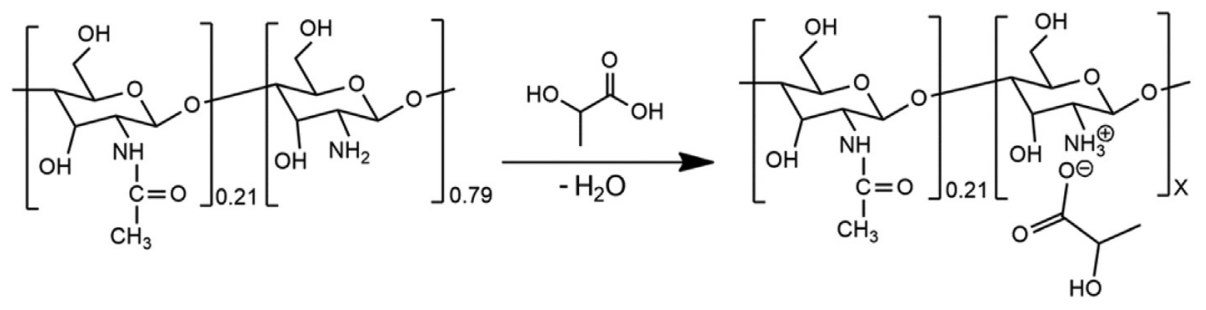

Fig. 5. Formation of the chitosan lactate intermediary in the formation of the amide bond.
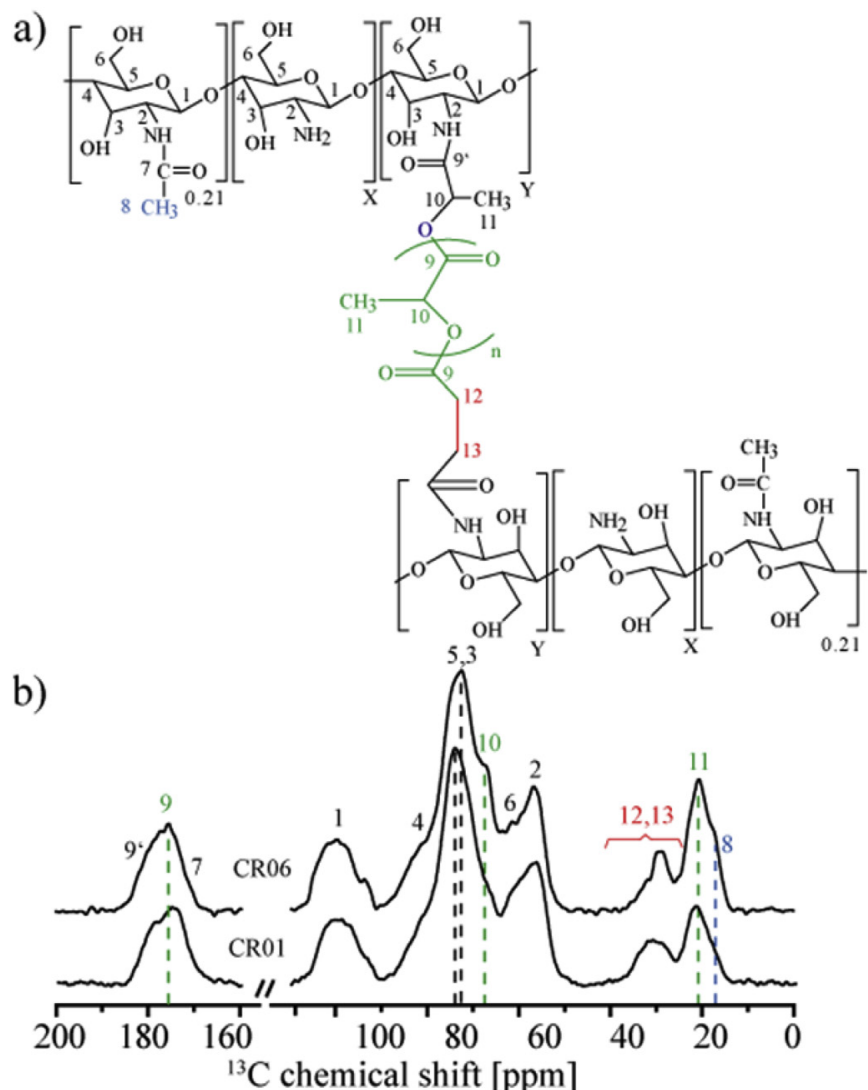

Fig. 6. a) Sketch of the basic unit of the CR samples. b) Comparison between MultiCP spectra of CR01 and CR06 samples. To improve the visualization, the region from 110 to $160 \mathrm{ppm}$ was removed, since only spinning sideband of $C=0$ groups are present. Note that at $4.5 \mathrm{kHz}$ spinning frequency only the $\mathrm{C}=0$ groups present side bands, which legitimate the quantification using the signals of the other groups.

succinyl units and the area under both succinyl signals, one obtains the percentage of crosslinked succinyl units. The values obtained for CR01, CR02, CR03, CR04, CR05 and CR06 were, respectively, $14 \%$, $23 \%, 32 \%, 36 \%, 38 \%$ and $53 \%$, with an error lower than $5 \%$. The graph of Fig. 7c illustrates these results.

\subsection{Dynamic mechanical analysis}

The $\tan \delta$ traces of the networks are shown in Fig. 8 and the corresponding values are listed in Table 2 . The main features of the DMA traces include the presence of a transition ranging from $~ 160$ to $\sim 180{ }^{\circ} \mathrm{C}$ that dominates the thermogram. The observed temperature values are significant lower than those corresponding to the lower temperature transitions and glass transition, observed for pure chitosan, above $300{ }^{\circ} \mathrm{C}$. This finding may be attributed to the separation of the chitosan chains by OLA units, which were held together by strong hydrogen bonds in the starting material. However, with the increase in crosslinking density, this number tends to increase up to $\sim 180^{\circ} \mathrm{C}$, in CR03. This progressive increase may be understood as a result of the "anchoring effect" (in the sense of hindering motions) due to crosslinks with short inserted OLA chains. Interestingly, a low temperature shoulder is clearly observed in the DMA traces. This shoulder may be related to relaxation (motions) of dangling OLA chains, which were not reacted to form crosslinks. For CR04, CR05 and CR06 the temperature of the main transition progressively decreases and the separation between the two observed transitions becomes less evident. This might be attributed to the further increases in the length of the OLA segments, turning this compound the major component of the system. Since a clear-cut phase separation is not expected in this crosslinked, high viscosity environment, the observed broad transitions represent a compromise between the predominant OLA phase and the chitosan phase, probably containing some residual OLA. This assumption holds also in relation to the small transitions seen at lower temperatures, as broad shoulders may represent the motion of OLA rich domains of large heterogeneity. Based on this interpretation, the crosslinked system behaves as a homogeneous material up to a certain amount of OLA incorporated, and undergoes phase separation, when the OLA content surpasses that level. The assumption of an OLA main phase when this compound is present in a large amount is corroborated by the tensile tests as shown next. It is noteworthy that the homopolymer, poly(DL-lactic acid) of high molecular mass is a stiff material and its inclusion in other systems could lead to a stiffening or brittleness even higher. In the present case however, the short oligo segments inserted served to separate the long backbones of chitosan, interrupting the hydrogen bonds responsible to its stiffness, imparting more free volume. The intermolecular forces operating in the high molecular mass poly(DL-lactic acid) are no longer present, at least not predominantly, in the OLA containing networks. Another remark that is worth mentioning is that the crosslinking is accompanied by an increase in the length of the inserted segments thus imparting them a certain degree of freedom that would allow for phase separation as found for other crosslinked materials [31]. The composition and transition temperatures of the networks are summarized in Table 2. For comparison purposes glass transition temperatures of pure OLA segments were determined previously [15] and ranged from $-5{ }^{\circ} \mathrm{C}$ to $30^{\circ} \mathrm{C}$ (molar masses from 1000 to 2500). Nevertheless no transitions in that range were detectable, in the DMA traces. Several factors could be accounted for the no detection of such relaxations, such as the fact that the short OLA segments were tied to the chitosan chain, hindering their motions. Additionally, ${ }^{1} \mathrm{H}$ MSE refocused FIDs were acquired as a function of temperature for all CR samples, and showed no transitions. The corresponding graphs can be viewed in Supplementary data.

\subsection{Mechanical test}

The mechanical tests were performed on films (thickness in the 



c)

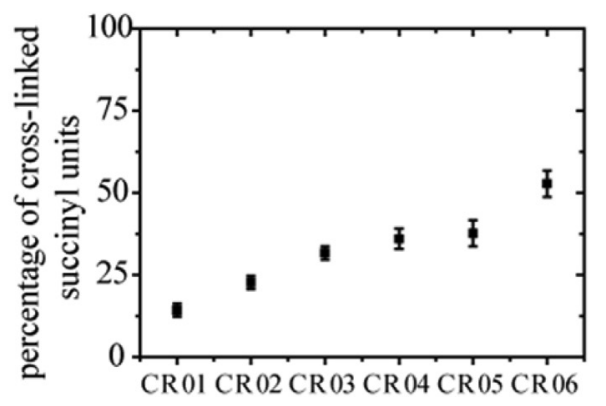

Fig. 7. a) MultiCP spectra of all CR samples in the region from 120 to $0 \mathrm{ppm}$ b) Example of spectral deconvolution of the $10-40 \mathrm{ppm}$ spectral region for sample CR04. c) Percentage of crosslinked succinyl units in CR samples.

range of $300 \mu \mathrm{m}$ ). The results obtained, shown in Table 2, agree very well with the transitions measured by DMA. Going from pure chitosan to CR01 (OLA 24.2\%; percentage of crosslinked succinyl units of $14 \%$ ) a decrease in strength properties is observed, due to the rupture of $\mathrm{H}$ bonds of the original chitosan. From CR02 (OLA 40.5\%, percentage of crosslinked succinyl units of 23\%) to CR04 (OLA $68.2 \%$, percentage of crosslinked succinyl units of $36 \%$ ) the tensile

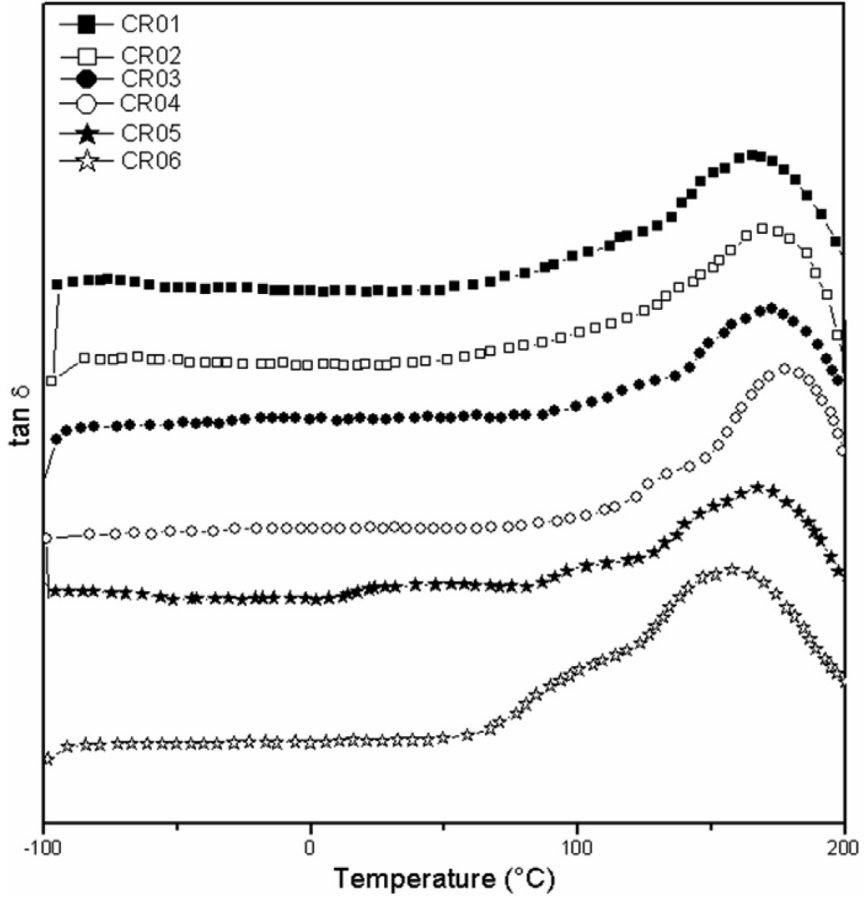

Fig. 8. $\tan \delta$ profiles of the networks.

properties increase indicating the formation of a more rigid material due to the anchoring effect of the crosslinking. CR04 showed the highest modulus, ultimate stress and $\mathrm{Tg}$, and the lowest elongation at break. With increases in the OLA content in CR05 (OLA 75.7\% percentage of crosslinked succinyl units of 38\%) and CR06 (OLA $80.8 \%$ percentage of crosslinked succinyl units of 53\%) an abrupt decay in modulus of elasticity and ultimate stress combined with the sharp increase in ultimate strain (maximum elongation) was observed, in accordance with the decrease in $\mathrm{Tg}$ of these materials. This finding supports the assumption of the formation of an OLA matrix with dispersed chitosan-rich domains. Oligo(DL-lactic acid) is more extensible than chitosan, and with increases in its content, the system acquires more mobility, with the separation of the chitosan chains.

The observed ductility increase or decrease with increasing crosslinking can be interpreted as follows: taking into account that the greater the number of interconnections the greater the length of OLA segments, the interplay of two effects is operating: one is the breaking of the chitosan hydrogen bonds leading to ductility and the other is the tying up of the chains leading to rigidity. The former (increased ductility) predominates in samples going from pure chitosan to CR01, and from CR04 to CR06 (longer OLA chains, less remaining hydrogen bonds), whereas the latter (rigidity increases) prevails going from CR01 to CR04 (shorter and more separated OLA segments, more remaining hydrogen bonds).

Additionally, another factor is noteworthy, which is the probable phase inversion, as noted. This effect operates in the same direction as the interplay above discussed.

In a previous work [15], the two materials used here to form networks were explored in two other combinations: blends and grafts. The blending resulted in very poor mechanical properties, did not afford useful materials in any proportion of the components, showing coarse phase separation as revealed by scanning microscopy. Several lengths of the OLA segments were tested, and even the short ones failed to provide a miscible or compatible blend. On the other hand, when covalently bonded in a grafted 
Table 2

Composition and transition temperatures of the networks. The values between brackets represent very small, broad, or shoulders in the curves.

\begin{tabular}{|c|c|c|c|c|c|c|c|}
\hline $\begin{array}{l}\text { Sample } \\
\text { labeling }\end{array}$ & $\begin{array}{l}\text { Percentage of crosslinking } \\
\text { succinyl units }^{\mathrm{a}}\end{array}$ & $\begin{array}{l}\text { Number average degree of } \\
\text { polymerization of OLA }\end{array}$ & 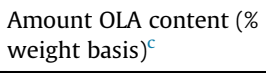 & $\begin{array}{l}\text { Transition } \\
\text { temperatures }{ }^{\mathrm{d}}\left({ }^{0} \mathrm{C}\right)\end{array}$ & $\begin{array}{l}\text { Elasticity } \\
\text { modulus (MPa) }\end{array}$ & $\begin{array}{l}\text { Ultimate } \\
\text { strain (\%) }\end{array}$ & $\begin{array}{l}\text { Ultimate } \\
\text { stress (MPa) }\end{array}$ \\
\hline CROO & $0 \pm 5$ & 0 & 0 & $>300$ & $2460 \pm 5$ & $6 \pm 1$ & $71 \pm 3$ \\
\hline CR01 & $14 \pm 5$ & 16.9 & 24.2 & 165.3 & $1600 \pm 100$ & $10 \pm 2$ & $43 \pm 6$ \\
\hline CRO2 & $23 \pm 5$ & 21.6 & 40.5 & 169.1 & $1700 \pm 100$ & $7 \pm 1$ & $46 \pm 5$ \\
\hline CR03 & $32 \pm 5$ & 31.2 & 57.4 & [129.5]; 172.3 & $1900 \pm 200$ & $7 \pm 2$ & $48 \pm 6$ \\
\hline CR04 & $36 \pm 5$ & 44.2 & 68.2 & [141.7]; 177.8 & $2100 \pm 300$ & $5 \pm 3$ & $55 \pm 9$ \\
\hline CR05 & $38 \pm 5$ & 60.6 & 75.7 & $\begin{array}{l}{[21.7] ;[116.2]} \\
167.1\end{array}$ & $1100 \pm 100$ & $15 \pm 2$ & $38 \pm 8$ \\
\hline CR06 & $53 \pm 5$ & 58.7 & 80.8 & {$[102.3] ; 157.8$} & $1000 \pm 100$ & $17 \pm 2$ & $32 \pm 7$ \\
\hline
\end{tabular}

${ }^{\text {a }}$ Number of crosslinking sites/100 repeating units of chitosan, determined by SSNMR.

b Stands for the length of the OLA segments.

c $\mathrm{g}$ of OLA/100 g sample.

d From DMTA measurements.

configuration (chitosan-g-oligo DL-lactic acid) the materials presented interesting properties, which varied according to the ratio between the two macromolecular species involved [15]. Nevertheless, these properties did not followed the trend described so far for the networks. In Figs. 9 and 10 the results regarding the thermal and mechanical behavior of the grafts were compared with those obtained here of the same composition, aiming to draw a comparison between the two classes of materials. The results can be summarized as follows: In a general way the mechanical and thermal properties of systems with the same composition but with different macromolecular architecture showed quite opposite trends.

Whereas Tg and moduli of the grafted copolymers continuously decay to a certain point with the increase of the degree of insertion and length of the pendant segments [15], the same properties in the

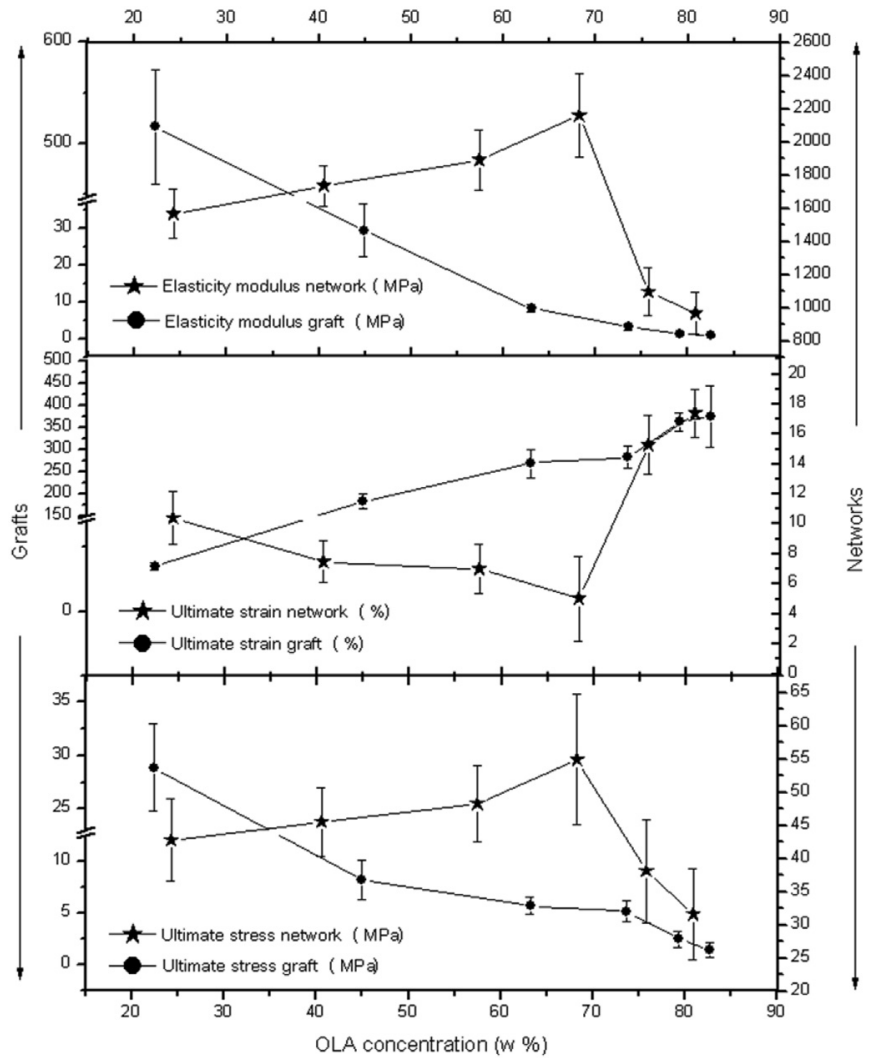

Fig. 9. Comparison between mechanical properties and composition for the grafted copolymers and networks. The data for the grafts were taken from previous work [15].

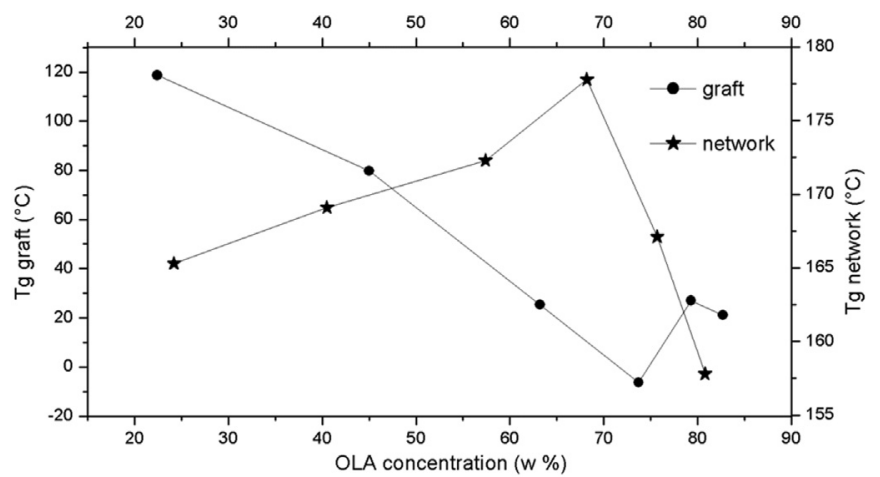

Fig. 10. Correlations between glass transition temperature and the OLA content for the graft copolymers and the networks.

networks increase to a very close point, which is located at the 70\% range of OLA content. From that point, the Tg of the grafts increases, probably due to an inversion of phase where the oligo(DL-lactic acid) predominates. Quite the opposite was observed for the networks; the Tg begins to decrease because the larger the length of the interchain segments the less the anchoring effect operates, reaching a point in which the free volume permits a large range of segmental mobility. A direct comparison of the Tg measurements with those related to the mechanical properties leads to similar reasoning, the transition (or inflexion) range is about the same, around the same composition range (70\% OLA content). It is noteworthy that only one Tg was found for all materials, but the $\tan \delta$ trace was characterized by a broad relaxation and might well encompass transitions for different phases, and certainly reflects a broad conformational dispersion. Nevertheless, the behavior of the glass transition can be taken as a gauge for the flexibility achieved with the progressive separation of the rigid chitosan chains. The differences in the profile of mechanical properties of the mentioned materials are a remarkable example of the influence of the configuration upon the macroscopic behavior.

Finally, the results show that the different configurations with their respective properties according to the ratio between components provide a means to design materials with a desired profile of mechanical and thermal behavior, using the procedures described and the graphs of Figs. 9 and 10 as starting points.

The composed products either as grafts or networks present lower moduli than the starting chitosan, but at the same time were much more soluble and pliable materials. The films were transparent and homogeneous and certainly with more potential to be applied as useful materials. 


\section{Conclusions}

The comparison between grafted copolymers and networks made up with chitosan and oligo(DL-lactic acid) revealed striking differences in thermal and mechanical behavior, for the same composition ratio of the two components. The degree of insertion of the grafted segments varied from 13.6 to 79.7/100 repeating units of chitosan and their average molar mass ranged from 300 to $1000 \mathrm{~g} \mathrm{~mol}^{-1}$. The number of crosslinking sites in the networks varied from 14 to $53 \%$ (crosslinking density) and the average molar mass of the segments from 1522 to $5287 \mathrm{~g} \mathrm{~mol}^{-1}$. Around $70 \%$ of incorporation of the oligo(DL-lactic acid), a morphological change took place and the observed behavior for both grafts and networks changed their trends in relation to the glass transition temperature and mechanical properties. Only one thermal relaxation was detected, but its broadness suggested that more than one phase was possible, enveloped in a dispersion of degrees of mixture. The results reported opened a way to design materials with a set of desired properties, without the need to prepare and compare all of them in advance.

\section{Acknowledgments}

The authors wish to acknowledge the financial support of the Brazilian Agencies (CNPq and CAPES) and the Institute of Organic Electronics (INEO).

\section{Appendix A. Supplementary data}

Supplementary data related to this article can be found at http:// dx.doi.org/10.1016/j.polymer.2016.04.031.

\section{References}

[1] C. Ninh, M. Cramer, C.J. Bettinger, Biomaterials Sci. 2 (5) (2014) 766-774. [2] S. Seuss, A.R. Boccaccin, Biomacromolecules 14 (10) (2013) 3355-3369.

[3] D.B. Khadka, D.T. Haynie, Nanomedicine Nanotechnol. Biol. Med. 8 (8) (2012)
$1242-1262$

[4] B. Fan, Y. Xing, Y. Zheng, C. Sun, G.X. Liang, Drug Deliv. 23 (1) (2016) 238-247.

[5] O. Garcia-Valdez, S. George, R. Champagne-Hartley, E. Saldivar-Guerra, P. Champagne, M.F. Cunningham, Polymer 67 (2015) 139-147.

[6] I. Safir, K.X. Ngo, J.N. Abraham, M.G. Afshar, E. Pavlova, C. Nardin, Polymer 79 (2015) 29-36.

[7] D. Dehnad, Z. Emarn-Djomeh, H. Mirzaei, S.M. Jafari, S. Dadashi, Carbohydr Polym. 105 (2014) 222-228.

[8] G.F. Perotti, J. Tronto, M.A. Bizeto, C.M.S. Izumi, M.L.A. Temperini, A.B. Lugao, D.F. Parra, V.R.L. Constantino, J. Braz. Chem. Soc. 25 (2) (2014) 320-330.

[9] M. Wihodo, C.I. Moraru, J. Food Eng. 114 (3) (2013) 292-302.

[10] A. Pourjavadi, Z.M. Tehrani, S. Jokar, Polymer 76 (2015) 52-61.

[11] S. Abbasi, G. Yousefi, O. Firuzi, S. Mohammadi-Samani, J. Appl. Polym. Sci. 133 (13) (2016).

[12] M. Matsui, M. Munaro, L. Akcelrud, Polym. Int. 59 (8) (2010) 1090-1098.

[13] M. Matsui, M. Munaro, L.C. Akcelrud, J. Polym. Res. 18 (6) (2011) 2255-2264.

[14] M. Matsui, L. Ono, L. Akcelrud, Polym. Test. 31 (1) (2012) 191-196.

[15] C.B. Ciulik, O.D. Bernardinelli, D.T. Balogh, E.R. de Azevedo, L. Akcelrud Polymer 55 (11) (2014) 2645-2651.

[16] R. Yamaguchi, Y. Arai, T. Itoh, S. Hirano, Carbohydr. Res. 88 (1) (1981) $172-175$.

[17] R.L. Johnson, K. Schmidt-Rohr, J. Magn. Reson. 239 (2014) 44-49.

[18] M. Kumar, React. Funct. Polym. 46 (1) (2000) 1-27.

[19] M. Rinaudo, Prog. Polym. Sci. 31 (7) (2006) 603-632.

[20] L. Zavaleta-Avejar, E. Bosquez-Molina, M. Gimeno, J.P. Perez-Orozco, K. Shirai Food Hydrocoll. 39 (2014) 113-119.

[21] Y.J. Han, K.H. Wang, J.Y. Lai, Y.L. Liu, J. Membr. Sci. 463 (2014) 17-23.

[22] M.E.I. Badawy, E.I. Rabea, N.E.M. Taktak, Carbohydr. Polym. 111 (2014) 670-682.

[23] Y. Xiao, T. Gong, Y. Jiang, Y.P. Wang, Z.Z.T. Wen, S.B. Zhou, C.Y. Bao, X.M. Xu, Polymer 82 (2016) $1-10$

[24] E.M. Krall, T.W. Klein, R.J. Andersen, A.J. Nett, R.W. Glasgow, D.S. Reader, B.C. Dauphinais, S.P. Mc Ilrath, A.A. Fischer, M.J. Carney, D.J. Hudson, N.J. Robertson, Chem. Commun. 50 (38) (2014) 4884-4887.

[25] J. Fernandez, A. Etxeberria, J.R. Sarasua, Polym. Degrad. Stab. 98 (7) (2013) 1293-1299.

[26] A.P. Zhu, T. Chen, L.H. Yuan, H. Wu, P. Lu, Carbohydr. Polym. 66 (2) (2006) $274-279$.

[27] J.R. Oliveira, M.C.L. Martins, L. Mafra, P. Comes, Carbohydr. Polym. 87 (1) (2012) 240-249.

[28] E.R. DeAzevedo, T.J. Bonagamba, D. Reichert, Prog. Nucl. Magn. Reson. Spectrosc. 47 (3-4) (2005) 137-164.

[29] K. Takegoshi, K. Hikichi, J. Chem. Phys. 94 (4) (1991) 3200-3206.

[30] A.E. Tonelli, NMR Spectroscopy and Polymer Microstructure: the Conformational Connection, Wiley, New York, 1989.

[31] S.H. Wang, S. Zawadzki, L. Akcelrud, J. Polym. Sci. Part B Polymer Phys. 38 (22) (2000) 2861-2872. 